\title{
Effect of Educational Program about Infection Control Precautions for Nurses in Pediatric
} Hemodialysis Units

Sadia Mohamed Ahmed ${ }^{1}$, Afkar Ragab Mohamed ${ }^{2}$, Sanaa Mahmoud Ahmed ${ }^{3}$

\author{
1- Clinical instructor- Technical Institute of Nursing - Minia University \\ 2- Professor of Pediatric Nursing - Faculty of nursing - Cairo University \\ 3- Assistant Professor of Pediatric Nursing - Faculty of Nursing- Minia University
}

\begin{abstract}
Background: Hemodialysis places children at high risk for infection because of patient comorbidities and numerous human, environmental, and procedural factors. The aim of this study was to evaluate the effect of the educational program on the nurse's knowledge and their practices about infection control measures in hemodialysis units. Research design: Pre /post quasi experimental design was used in this study. Sample: A convenient sample of all nurses in hemodialysis units, Setting: the study was conducted in pediatric hemodialysis units at Minia University and Minia General Hospitals. Tools for Data Collection: A structured interview questionnaire sheet in Arabic language was used for the studied nurses as pre/posttest. It composed of the following parts: Part I: Demographic data related to the nurses' age, sex, qualification, years of experience and previous attending courses in infection control. Part II: Nurses, knowledge related to infection control in pediatric hemodialysis unites Part III: An observational checklist that was adopted from Control Diseases Center. (National Center for emerging and zoonotic infectious diseases division of health quality promotion). Results: the finding of the current study revealed that there were statistical significant differences between nurses' knowledge and practices at pre and post- program. There were significance correlations between nurses' knowledge, residence and occupation respectively at pre- program. Conclusion the study concluded that there were statistical significant differences between nurses' knowledge and practices at pre and post- program. Recommendation the study recommended that the developed program should be applied and repeat again every 6 months as well as provision of continuing education programs on regular basis is suggested in order to refresh and update nurse's knowledge.
\end{abstract}

KeyWords: Infection Control, Hemodialysis, Pediatric nurse, chronic kidney disease

\section{Introduction}

Hemodialysis remained the most common treatment modality with approximately 1,929,000 patients undergoing hemodialysis, for $89 \%$ of all dialysis patients In Egypt the total prevalence of patients on dialysis is 264 per million (CDC,2014). Nurses are directly responsible for patients undergoing hemodialysis and they administer care to reduce the complications (Jane \& Ruth, 2012).

The patient and the dialysis apparatus should be under supervision of nurses consistently, so that different potential complications can be detected. Dialysis nurses must have knowledge and skills, because they considered important features of quality nursing care in the hemodialysis (Judith\& Karen, (2013).

Hemodialysis places children at high risk for infection because of patient comorbidities and numerous human, environmental, and procedural factors. Establishing an infection prevention and control program which includes a bundle of strategies and interventions that are consistently performed will reduce the infection risk for both employees and patients (Hassona, Winkelman, El-Wahab, Ali \& Abdeen, (2012).

Patients who undergo hemodialysis have a higher risk of infection, due to the following factors: Frequent use of catheters or insertion of needles to access the bloodstream, weakened immune systems, frequent hospital stays and prolonged hospital stay, and increased medical treatment costs. Infectious complications are a serious clinical problem and they are associated with high rates of morbidity and mortality (Elsayed, El-Soreety, Elawany \& Nasar,2012).

Infection is reported as the second most common cause of death in hemodialysis patients (20.2\%), after cardiovascular disease. by the United States National Nosocomial Infection Surveillance, the admission rate for pneumonia rose $7.3 \%$, for bacteremia/septicemia $31 \%$, and for cellulitis $20.3 \%$. There were 103 admissions per 1000

$P$ a g e $\mid 77$ patient years with the diagnosis of bacteremia/septicemia and 129 per 1000 patient years with the diagnosis of vascular access-associated infection (Judith, Guzman \& Karen, 2013).

Infection prevention is essential to understand the transmission of a pathogen resulting in colonization or infection requires the following six vital links, causative agent infectious reservoir, portal of exit from the reservoir, mode of transmission, portal of entry into host and susceptible host. Each link must be present for infection or colonization to proceed and breaking any of the links can prevent the infection. The aim of isolation precautions is to interrupt these links (CDC, 2011).

Recent guidelines for the prevention of transmission of infection published in the United States and Canada are based on the principles that certain precautions are required for the care of all patients, regardless of diagnoses, and are determined by the task performed and that further measures are required for patients with certain infections, and are determined by the route of transmission of the infection (CDC. 2010b).

The nurse is considering the first line to seek to improve clinical practice in the hemodialysis units. It was important to assess the ability of nurses to create positive change in the practice. The continuous advances in technology increased the complexity of hemodialysis treatment and put patients at a higher risk for infection due to several etiologies related to chronic disease (Abdelsatir, 2013).

Healthcare associated infections (HAIs) are preventable through Implementation of best infection prevention and control practices. This will facilitate the delivery of high quality health care for patients and a safe working environment for our healthcare workers. These national guidelines are developed to provide a coordinated approach to the prevention and management of HAIs (Abou El-Enein \&EL Mahdy,2011). 
Dialysis centers are not hospital-based and therefore they frequently do not have access to the trained infection perfectionists that provide infection control expertise in hospitals. Dialysis technicians, who provide a large amount of care in dialysis settings, generally receive limited formal training in infection control and aseptic technique (CDC, 2013).

Key Infection Prevention Practices: Perform hand hygiene frequently and change gloves, Maintain separate clean areas for supplies and medications and separate contaminated areas for used items, Practice proper handling and delivery of patient supplies and medications Perform effective cleaning and disinfection of dialysis equipment and environmental surfaces, Carefully handle medications and the patient's vascular access to avoid contamination (Chenoweth,Hines,Hall,Saran,Kalbfleisch, et al, 2015).

The role of the Nurse in Infection Prevention and Control Specialist is straight forward: identify, prevent, and control outbreaks of infection in health care settings and the community; however, the activities, knowledge and skills required are exceptionally advanced and complex. While the role is straight forward, the span of activities to meet the responsibilities of the role is broad, and the methods are varied (Emily\& Trish, 2011).

\section{Significance of the study}

Chronic kidney disease (CKD) is an emerging global public health problem. The disease is a component of a new epidemic of chronic conditions that replaced malnutrition and infection as leading causes of mortality during the 20th century (Ahamed \& Sallam, 2018)).

Age standardized death rates due to CKD have increased during the last 23 years. CKD has shifted from the 36th cause of death in 1990 to the 19th cause in 2013 (Association for professionals Infection center, (2014).

A change in the global approach to CKD from the treatment of end stage renal disease (ESRD) to intensive primary and secondary prevention is therefore considered an absolute public health priority(Abd ElHafeez,Bolignano,D'Arrigo, Dounousi, Tripepi, and Zoccali, 2018).

Infection is the most common cause of hospitalization in hemodialysis patients and has become the second major cause of death. Lack of hygiene by staff increases the chance of infection (E Bagheban, SLakdizaji \& Zamanzadeh, 2018).

The increasing risk of pediatric infections because of poor of infection control practices which causes significant children morbidity and mortality as well as increased hospitalization. pediatric patient admitted to hemodialysis unit as the last end stage of renal failure, routinely as two to three time at a weak. Are routinely subjected to many procedures that invades (enters) the body (Abdelsatir, 2013).

The prevalence of $\mathrm{HCV}$ infection among dialysis patients in developed countries ranges from 3.6 to $20 \%$, with higher rates in developing countries. It was estimated to be $52.1 \%$ in Egypt. There is a high prevalence of HCV among HD patient (Lotfy, Sakla\& El-Naggar ,2015).

So the pediatric nurses need theoretical and technical information about infection control precautions in hemodialysis units. There for developing and applying nursing intervention for nurses about infection control of some procedure in the hemodialysis units, is very important and beneficial in terms of quality of care (Mabrouke ,2015).

$P$ a g e $\mid 78$
Aim of the study

The aim of the current study was to evaluate the effect of educational program about infection control precautions for nurses in pediatric hemodialysis units; this is through the following.

1) Assess the nurses' knowledge and practices about infection control measures in the hemodialysis units before applying educational program.

2) Develop and apply an educational program about infection control precautions for nurses in pediatric hemodialysis units .

3) Evaluate the effect of the educational program on the nurses' knowledge and practices about infection control precautions in pediatric hemodialysis.

\section{Research hypothesis}

Pediatric hemodialysis nurses knowledge and practices' mean scours will increase about infection control precaution after receiving an educational program than before.

\section{Subjects and Methods}

Research design: Pre/post quasi experimental design was used in this study. A quasi experimental design is one type of experimental design that very similar to the true experimental design except there is loss one criteria which is control, manipulation or randomization (Burns \& Grove , 2012).

Research Setting: The study was conducted in pediatric hemodialysis units at Minia University and Minia General Hospitals.

Subject: A convenient sample of all nurses in hemodialysis units (36) nurses; from Minia University Hospital and Minia General Hospital.

Tools for Data Collection: Tool 1:- A structured interview questionnaire sheet in Arabic language was used for the studied nurses as pre/posttest. It was designed by the research investigator after extensive review of the related literature. It composed of the following parts.

- Part I- Demographic data related to the nurses' age, sex, qualification, years of experience and previous attending courses in infection control.

- $\quad$ Part II- Nurses, knowledge related to infection control in pediatric hemodialysis unites that involve 31 items of closed -ended questions were divided into knowledge about infection control in general, which consisted of 26 items and 5 items about infection in hemodialysis units, the total score of knowledge equal 93 degree.

- Part III:An observational checklists that was adopted from Control Diseases Center (CDC, 2014) National Center for emerging and zoonotic infectious diseases division of health quality promotion) to assess the nurses, practices about infection control procedures in hemodialysis unites which include (hand washing(10 steps), wearing gloves(5 steps), administration intramuscular injection (7 steps), hemodialysis injectable medication administration preparation ( 7 steps), hemodialysis injectable medication administration (8steps), arteriovenous (AV) fistula graft cannulation (10 steps) and AV fistula graft 
decannulation (8 steps), hemodialysis catheter connection ( 9 steps ) and hemodialysis catheter disconnection (10 steps), hemodialysis catheter exit site care (10 steps) and dialysis station routine disinfection before( 8 steps $)$ and after receiving patient ( 6 steps $)$.

The nurses' actual practices were assessed by the research investigator using the checklists by direct and indirect observation. The nurses, practices evaluated as done or not done where a score of 1 was given for done correctly, score of zero was given for not done practice these scores were converted in to a percentage score. The nurses, practice was considered satisfactory if the percent score is $60 \%$ or more and unsatisfactory if less than $60 \%$. It was done during actual routine work in the morning shift.

\section{Scoring system:}

For knowledge; the total score ( 92) was categorized into three levels unsatisfactory if score less $60 \%$, average for $60 \%-70 \%$, and good for more than $70 \%$. For practice; the total score (96) was categorized into two levels unsatisfactory if scoreless $60 \%$, and satisfactory for more than $60 \%$. For each part, the score of the items were summed up and the total divided by number of items, giving a mean score for the area. These scores were converted into a percent score and mean and standard deviations were computed. These scores were converted into a percent score. The nurse's practice was considered satisfactory if the percent score is $60 \%$ or more and unsatisfactory if scored less than $60 \%$ (Mohamed, 2018).

\section{Tool Validity and Reliability}

Data collection tools related to nurses practices were adopted from Control Diseases Center (CDC.2014) National Center for emerging and zoonotic infectious diseases division of health quality promotion) to assess the nurses, practices about infection control procedures in hemodialysis, the tools are valid and reliable.

\section{Pilot study}

A pilot study was carried out on (4) nurses from the selected units using the previously mentioned tools, to evaluate their applicability and clarity and to estimate time for each tool. According to results of the pilot study the necessary modification was done. Nurses involved in the pilot study were included from the main study subjects.

\section{The program}

The study work was conducted in the period from November 2016 to April 2017, the research investigator was available daily by rotation at each study setting, each group was given the freedom to choose their optimal time for receiving the program whenever they have minimal work load. Each session usually started by a summary of what has been taught during the preceding session. Giving praise / recognition to the studied nurses were used as motivation during program implementation.

The session started by meeting the nurses throughout the morning shift, the research investigator first introduced herself to them and gave them a complete background about the study, its aim, and then the pretest format was distributed in order to collect the required data. The research investigator was available for more clarification whenever needed. Then, the content of the program was designed based on actual educational need assessment of the studied nurses. Consequently, the subject content has been sequenced through theoretical and practical sessions.

Method of teaching involved modified lecture, group discussion, pictures, and data show on my personal computer and demonstration and re-demonstration. Suitable teaching aids were used especially for the program such as real equipment and facilities.

It was designed in Arabic form of educational program by the research investigator based upon the actual need assessment of nurses. It was also supplemented with information based on review of relevant literature (nursing textbook, journals and internet resources) about infection control in pediatric hemodialysis

\section{Data collection procedure}

Administrative approval was obtained from ethics committee; Faculty of Nursing, Minia University. An official permission was obtained from the concerned hospital authorities to conduct the study. The aim, and expected out comes of the study was explained to the administrators, as well as participants nurses.

Application of the program sessions was organized according to the time available to the nurses in each hemodialysis units, the time required for the program implementation was in six months, and the nurses will be interviewed two days /week from 9am: 12am. Nurses were divided in to nine small groups.

The program was conduct over two sessions per day to cover all information in knowledge and practices about infection control in hemodialysis. Different teaching methods were used to motivate and reinforce techniques. A copy of the program booklet was given to each participant. "Post-test" was carried out immediately and after six months after implementation of training program to assess the effect of the program on nurse's knowledge and practices about infection control in hemodialysis units.

The educational program for nurses working in hemodialysis unit included a guidance book which covered theoretical and Practical parts through the following items: Theoretical part included: definition of infection, cycle if infection, rout of transmission, high risk group and general precaution; Practical part: it composed of the application of procedures(hand washing, wearing gloves, administration intramuscular injection, hemodialysis injectable medication administration preparation, hemodialysis injectable medication administration, arterio-venous fistula graft cannulation and arterio-venous fistula graft decannulation, hemodialysis catheter connection and hemodialysis catheter disconnection, hemodialysis catheter exit site care and dialysis station routine disinfection before and after receiving patient.

\section{Ethical consideration}

The purpose of the study was explained to the nurses and oral consent was obtained from them to participate in this study. They were given an opportunity to withdraw from the study without given a reason and they were assured that anonymity and confidentiality of information was protected. Ethics, values, culture, and beliefs were respected.

\section{Statistical analysis}

Data entry was done using compatible personal computer. The statistical analysis was done using SPSS-19 Statistical Software Package. Data were presented using 
descriptive statistics in the form of frequencies and percentages for qualitative variables and mean and standard deviations for quantitative variables. The quantitative continuous data were compared by using student t-test in case of comparison between the mean scores of the study group. Qualitative studied variable were compared using chisquare test. Person correlation analysis used for assessment of the inter-relationships between the nurses knowledge and practices about infection control of procedure, statistical significance differences at value $<0.05$

\section{Results}

Table (1): Distribution of the Studied Nurses according To Their Demographics characteristic N=36

\begin{tabular}{|c|c|c|c|}
\hline \multirow{2}{*}{\multicolumn{2}{|c|}{ Nurses Data }} & \multicolumn{2}{|c|}{$\mathrm{N}=36$} \\
\hline & & No & $\%$ \\
\hline \multicolumn{4}{|c|}{ Age } \\
\hline & $20<30$ & 20 & 55.6 \\
\hline- & $30<40$ & 12 & 22.2 \\
\hline- & $\geq 40$ & 4 & 11.1 \\
\hline \multicolumn{4}{|c|}{ Sex } \\
\hline- & Male & 10 & 27.8 \\
\hline- & Female & 26 & 72.2 \\
\hline \multicolumn{4}{|c|}{ Years of experience: } \\
\hline & & 13 & 36.1 \\
\hline & & 13 & 36.1 \\
\hline & & 10 & 27.8 \\
\hline \multicolumn{4}{|c|}{ Qualification of nurses: } \\
\hline- & Diploma of Secondary Nursing School & 17 & 47.2 \\
\hline- & Bachelor in Nursing Sciences & 7 & 19.4 \\
\hline- & Technical Institute of nursing & 12 & 33.3 \\
\hline \multicolumn{4}{|c|}{ Occupation: } \\
\hline - & Technical & 30 & 83.3 \\
\hline- & Specialist & 6 & 16.7 \\
\hline \multicolumn{4}{|c|}{ Previous attendance of training courses: } \\
\hline \multicolumn{4}{|c|}{ Residents } \\
\hline- & Ruler & 19 & 52.8 \\
\hline- & Urban & 17 & 47.2 \\
\hline
\end{tabular}

Table (1) shows that more than half (55.6\%) of the studied nurses are between 20 to less than 30 years old, more than two thirds $(72.2 \%)$ of the studied nurses were female, while less than one third $(27.8 \%)$ were male. nearly one third of studied nurses (36.1\%) have less than five years of experience, less than half $(47.2 \%)$ of the them have diploma of secondary nursing school. Regarding to their job description the majority (83.3\%) are technical nurses. All the studied nurses (100\%) had previous attendance of training courses affiliated to (ICU), (EMOH) and (WHO), more than half of them $(52.8 \%)$ were from ruler area.

Table (2) Comparison of Nurses' Knowledge related to Infection Control Pre, Post and after 6 months of the educational Program (N-36)

\begin{tabular}{|c|c|c|c|c|c|c|c|c|}
\hline \multirow[t]{2}{*}{ Knowledge Related to Infection Control } & \multicolumn{2}{|c|}{$\begin{array}{c}\text { Pre } \\
\text { program }\end{array}$} & \multicolumn{2}{|c|}{ Post program } & \multicolumn{2}{|c|}{$\begin{array}{l}\text { After } 6 \\
\text { Months }\end{array}$} & \multirow[t]{2}{*}{$\chi^{2}$} & \multirow[t]{2}{*}{$\mathrm{P}$} \\
\hline & No & $\%$ & No & $\%$ & No & $\%$ & & \\
\hline $\begin{array}{l}\text { *Infection control } \\
\text { Poor } \\
\text { Average } \\
\text { Good }\end{array}$ & $\begin{array}{l}22 \\
7 \\
1\end{array}$ & $\begin{array}{l}77.8 \\
19.4 \\
2.8\end{array}$ & $\begin{array}{l}16 \\
14 \\
6\end{array}$ & $\begin{array}{l}44.4 \\
38.9 \\
16.7\end{array}$ & $\begin{array}{l}33 \\
2 \\
-\end{array}$ & $\begin{array}{l}91.7 \\
11.1 \\
-\end{array}$ & 22.5 & $0.001 *$ \\
\hline $\begin{array}{l}\text { *Hand washing } \\
\text { Poor } \\
\text { Average }\end{array}$ & $\begin{array}{l}16 \\
20\end{array}$ & $\begin{array}{l}44.4 \\
55.6\end{array}$ & $\begin{array}{l}6 \\
30\end{array}$ & $\begin{array}{l}16.7 \\
83.3\end{array}$ & $\begin{array}{l}26 \\
10\end{array}$ & $\begin{array}{l}72.2 \\
27.8\end{array}$ & 22.5 & $0.001 *$ \\
\hline $\begin{array}{l}* \text { General Protection measures } \\
\text { Poor } \\
\text { Average }\end{array}$ & $\begin{array}{l}35 \\
1\end{array}$ & $\begin{array}{l}97.2 \\
2.8\end{array}$ & $\begin{array}{l}16 \\
20\end{array}$ & $\begin{array}{l}44.4 \\
55.6\end{array}$ & $\begin{array}{l}27 \\
9\end{array}$ & $\begin{array}{l}75 \\
25\end{array}$ & 25.2 & $0.001 *$ \\
\hline $\begin{array}{l}\text { *Precaution in hemodialysis } \\
\text { Poor } \\
\text { Average } \\
\text { Good }\end{array}$ & $\begin{array}{l}22 \\
9 \\
5\end{array}$ & $\begin{array}{l}61.1 \\
25 \\
13.9\end{array}$ & $\begin{array}{l}35 \\
1\end{array}$ & $\begin{array}{l}97.2 \\
2.6\end{array}$ & $\begin{array}{l}28 \\
4 \\
4\end{array}$ & $\begin{array}{l}77.8 \\
11.1 \\
11.1\end{array}$ & 14.7 & $0.005 *$ \\
\hline $\begin{array}{l}\text { Total of nurses' knowledge about } \\
\text { infection control. } \\
\text { Poor }\end{array}$ & 26 & 72.2 & 17 & 47.2 & 32 & 88.9 & 18.1 & $0.001 *$ \\
\hline
\end{tabular}




\begin{tabular}{|c|c|c|c|c|c|c|c|c|}
\hline \multirow{2}{*}{ Knowledge Related to Infection Control } & \multicolumn{2}{|c|}{$\begin{array}{c}\text { Pre } \\
\text { program }\end{array}$} & \multicolumn{2}{|c|}{ Post program } & \multicolumn{2}{|c|}{$\begin{array}{l}\text { After } 6 \\
\text { Months }\end{array}$} & \multirow{2}{*}{$\chi^{2}$} & \multirow[t]{2}{*}{$\mathrm{P}$} \\
\hline & No & $\%$ & No & $\%$ & No & $\%$ & & \\
\hline $\begin{array}{l}\text { Average } \\
\text { Good }\end{array}$ & $\begin{array}{l}9 \\
1\end{array}$ & $\begin{array}{l}25 \\
2.8\end{array}$ & $\begin{array}{l}13 \\
6\end{array}$ & $\begin{array}{l}36.1 \\
17.7\end{array}$ & $\begin{array}{l}4 \\
0\end{array}$ & $\begin{array}{l}11.1 \\
0.00\end{array}$ & & \\
\hline
\end{tabular}

Significance $(\mathrm{p} .<0.05)$

It is clear from table 2 that there are statistical significance differences between pre, post and after 6 Months of the educational program, regarding the knowledge about infection control (p.0.001). So there are observed elevation in the knowledge level in post program than in preprogram in relation to hand washing, general precaution measures and precaution in hemodialysis. There is decrease in the knowledge level after six months of educational program.

Table (3): Comparison of Mean Scores of the Nurses' Practice Related to Universal Precaution of Infection Control Pre/Post Educational Program n= 36

\begin{tabular}{|l|l|l|l|l|}
\hline \multicolumn{1}{|c|}{ Items } & \multicolumn{2}{c|}{ practice } & \multirow{2}{*}{ t. test } & \multirow{2}{*}{ P. value } \\
\cline { 2 - 3 } & \multicolumn{1}{|c|}{ Pre program } & \multicolumn{1}{|c|}{ Post program } & \multicolumn{2}{l}{} \\
\hline Hand washing & $18.3 \pm 3.8$ & $30.4 \pm 3.7$ & 13.425 & $0.001^{*}$ \\
\hline Wearing gloves & $7.5 \pm 2.1$ & $13.9 \pm 2.1$ & 12.820 & $0.001^{*}$ \\
\hline Administration of intramuscular injection & $12.7 \pm 1.6$ & $19.8 \pm 3.5$ & 10.775 & $0.001^{*}$ \\
\hline $\begin{array}{l}\text { Hemodialysis injectable medication } \\
\text { administration preparation }\end{array}$ & $13.3 \pm 2.3$ & $25.1 \pm 4.4$ & 14.053 & $0.001^{*}$ \\
\hline $\begin{array}{l}\text { Hemodialysis injectable medication } \\
\text { administration }\end{array}$ & $11.7 \pm 2.3$ & $22.4 \pm 4.1$ & 13.275 & $0.001^{*}$ \\
\hline Total of nurses' practice about infection control. & $63.7 \pm 8.7$ & $99.1 \pm 17.3$ & 14.859 & $0.001^{*}$ \\
\hline
\end{tabular}

In comparison of means scores of the studied nurses' practice related to universal precaution table (3) illustrates that there are highly statistical significant differences between nurses' practice at pre and post- program (p. $<0.05)$. Regarding to hand washing, wearing gloves, administration of intramuscular injection, hemodialysis injectable medication administration preparation and hemodialysis injectable medication administration.

Table (4): Comparison of Mean Scores of the Nurses' Practice Related to Universal Precaution of Infection Control Pre/after six months Educational Program n= 36

\begin{tabular}{|l|l|l|l|l|}
\hline \multirow{2}{*}{ Items } & \multicolumn{2}{|c|}{ practice } & \multirow{2}{*}{ t. test } & \multirow{2}{*}{ P. value } \\
\cline { 2 - 4 } & \multicolumn{1}{|c|}{ Pre program } & After six months & & \\
\hline Hand washing & $18.3 \pm 3.8$ & $30.4 \pm 3.7$ & 5.868 & $0.001^{*}$ \\
\hline Wearing gloves & $7.5 \pm 2.1$ & $13.9 \pm 2.1$ & 4.206 & $0.001^{*}$ \\
\hline $\begin{array}{l}\text { Administration of intramuscular } \\
\text { injection }\end{array}$ & $12.7 \pm 1.6$ & $19.8 \pm 3.5$ & 4.151 & $0.001^{*}$ \\
\hline $\begin{array}{l}\text { Hemodialysis injectable medication } \\
\text { administration preparation }\end{array}$ & $13.3 \pm 2.3$ & $25.1 \pm 4.4$ & 5.368 & $0.001^{*}$ \\
\hline $\begin{array}{l}\text { Hemodialysis injectable medication } \\
\text { administration }\end{array}$ & $11.7 \pm 2.3$ & $22.4 \pm 4.1$ & 6.960 & $0.001^{*}$ \\
\hline $\begin{array}{l}\text { Total of nurses' practice about infection } \\
\text { control. }\end{array}$ & $63.7 \pm 8.7$ & $99.1 \pm 17.3$ & 7.600 & $0.001^{*}$ \\
\hline
\end{tabular}

In comparison of means scores of the studied nurses' practice related to universal precaution table (4) illustrates that there are highly statistical significant differences between nurses' practice at pre and after six months of program (p.<0.05). Regarding to hand washing, wearing gloves, administration of intramuscular injection, hemodialysis injectable medication administration preparation and hemodialysis injectable medication administration.

Table: (5) Comparison of Mean Scores of the Nurses' Practice Related to Hemodialysis Procedure Pre/Post Educational Program $N=36$

\begin{tabular}{|l|l|l|l|l|}
\hline \multirow{2}{*}{ Items } & \multicolumn{2}{c|}{ Practice } & \multirow{2}{*}{ t. test } & \multirow{2}{*}{ P. value } \\
\cline { 2 - 4 } & \multicolumn{1}{c|}{ Pre program } & Post program & & \\
\hline Hemodialysis injectable medication administration & $11.7 \pm 2.3$ & $22.4 \pm 4.1$ & 14.053 & $0.001^{*}$ \\
\hline Arteriovenous fistula graft cannulation & $13.3 \pm 2.3$ & $25.1 \pm 4.4$ & 13.275 & $0.001^{*}$ \\
\hline Arteriovenous fistula graft decannulation & $11.7 \pm 2.3$ & $22.4 \pm 4.1$ & 13.995 & $0.001^{*}$ \\
\hline Hemodialysis catheter connection & $13.8 \pm 2.1$ & $25.4 \pm 4.4$ & 14.940 & $0.001^{*}$ \\
\hline Hemodialysis catheter disconnection & $15.7 \pm 1.8$ & $28.2 \pm 4.6$ & 14.645 & $0.001^{*}$ \\
\hline Hemodialysis catheter exit site care & $14.6 \pm 2.3$ & $28.1 \pm 5.02$ & 13.658 & $0.001^{*}$ \\
\hline Dialysis Station Routine Disinfection & $25.6 \pm 3.1$ & $37.5 \pm 4.2$ & 14.669 & $0.001^{*}$ \\
\hline
\end{tabular}




\begin{tabular}{|l|l|l|l|l|}
\hline \multicolumn{1}{|c|}{ Items } & \multicolumn{2}{|c|}{ Practice } & \multirow{2}{*}{ t. test } & \multirow{2}{*}{ P. value } \\
\cline { 2 - 4 } & Pre program & Post program & & \\
\hline $\begin{array}{l}\text { Total of nurses' practice about hemodialysis } \\
\text { procedure. }\end{array}$ & $94.9 \pm 11.9$ & $167 \pm 26.9$ & 14.7 & $0.001^{*}$ \\
\hline
\end{tabular}

In comparison of means scores of the studied nurses' practice related to hemodialysis procedure table (5) illustrates that there are highly statistical significant differences between nurses' practices related to hemodialysis procedure at pre and posteducational program ( $\mathrm{p} .<0.05)$. Regarding to hemodialysis injectable medication administration, arteriovenous fistula graft cannulation, arteriovenous fistula graft decannulation, hemodialysis catheter connection, hemodialysis catheter disconnection, and hemodialysis catheter exit site care and dialysis station routine disinfection.

Table: (6) Comparison of Mean Scores of the Nurses' Practice Related to Hemodialysis Procedure Pre/after six months of Educational Program N=36

\begin{tabular}{|l|l|l|l|l|}
\hline \multicolumn{1}{|c|}{ Items } & \multicolumn{2}{c|}{ Practice } & \multirow{2}{*}{ t. test } & \multirow{2}{*}{ P. value } \\
\cline { 2 - 3 } & \multicolumn{1}{|c|}{ Pre program } & \multicolumn{1}{|c|}{ after 6months } & & \multirow{2}{*}{$0.001^{*}$} \\
\cline { 1 - 4 } $\begin{array}{l}\text { Hemodialysis injectable medication } \\
\text { administration }\end{array}$ & $11.7 \pm 2.3$ & $17.3 \pm 4.1$ & 6.960 & $0.001^{*}$ \\
\hline Arteriovenous fistula graft cannulation & $13.3 \pm 2.3$ & $19.2 \pm 6.1$ & 5.368 & $0.001^{*}$ \\
\hline Arteriovenous fistula graft decannulation & $11.7 \pm 2.3$ & $17.3 \pm 4.1$ & 6.960 & $0.001^{*}$ \\
\hline Hemodialysis catheter connection & $13.8 \pm 2.1$ & $19.2 \pm 4.4$ & 6.480 & $0.001^{*}$ \\
\hline Hemodialysis catheter disconnection & $15.7 \pm 1.8$ & $20.5 \pm 4.2$ & 6.223 & 0.5 \\
\hline Hemodialysis catheter exit site care & $14.6 \pm 2.3$ & $19.5 \pm 4.1$ & .661 & $0.001^{*}$ \\
\hline Dialysis Station Routine Disinfection & $25.6 \pm 3.1$ & $25.1 \pm 3.9$ & 7.246 & $0.001^{*}$ \\
\hline $\begin{array}{l}\text { Total of nurses' practice about hemodialysis } \\
\text { procedure. }\end{array}$ & $94.9 \pm 11.9$ & $120.9 \pm 17.9$ & 7.2 & \\
\hline
\end{tabular}

In comparison of means scores of the studied nurses' practice related to hemodialysis procedure table (6) illustrates that there are highly statistical significant differences between nurses' practices related to hemodialysis procedure at pre and after six months of educational program $(\mathrm{p} .<0.05)$. Regarding to hemodialysis injectable medication administration, arteriovenous fistula graft cannulation, arteriovenous fistula graft decannulation, hemodialysis catheter connection, hemodialysis catheter disconnection, and dialysis station routine disinfection. While no statistical significant differences regarding hemodialysis catheter exit site care (p.>0.05).

Table (7): Correlation between Demographic Characteristic and Total Knowledge among the studied nurses.

\begin{tabular}{|l|l|l|l|l|l|l|}
\hline \multirow{2}{*}{\multicolumn{1}{|c|}{ Demographic data }} & \multicolumn{6}{|c|}{ Total knowledge correlation } \\
\cline { 2 - 7 } & \multicolumn{2}{|c|}{ Pre program } & \multicolumn{2}{c|}{ Post program } & \multicolumn{2}{c|}{ After 6 months } \\
\cline { 2 - 7 } & $\mathrm{R}$ & $\mathrm{P}$ & $\mathrm{R}$ & $\mathrm{P}$ & $\mathrm{R}$ & $\mathrm{P}$ \\
\hline Sex & -0.06 & 0.7 & -0.07 & 0.6 & -0.23 & 0.1 \\
\hline Residents & -0.47 & $0.001^{*}$ & -0.21 & 0.2 & -0.25 & 0.1 \\
\hline Age & -0.004 & 0.9 & -0.01 & 0.9 & -0.02 & 0.9 \\
\hline Years of experience & -0.007 & 0.9 & -0.15 & 0.3 & -0.14 & 0.3 \\
\hline Qualification of nurses & -0.24 & 0.1 & 0.06 & 0.7 & 0.14 & 0.4 \\
\hline Occupation & 0.35 & $0.03^{*}$ & 0.36 & $0.02^{*}$ & 0.06 & 0.6 \\
\hline
\end{tabular}

Table (7) shows that there are no statistical significance correlation between demographic characteristics and total knowledge among the studied nurses at pre, post and after 6 months of the educational program. While regarding occupation there is mild significance correlation (r. 0.36) at post education program. There are significance correlation between nurses' knowledge, residence and occupation (p. $0.001 \& 0.03$ ) respectively at pre- program.

Table (8): Correlation between Demographic Characteristic and Total Infection Control Practice among the Studied Nurses.

\begin{tabular}{|l|l|l|l|l|l|l|}
\hline \multirow{2}{*}{} & \multicolumn{6}{|c|}{ Total infection control practice correlation } \\
\cline { 2 - 7 } & \multicolumn{2}{|c|}{ Pre program } & \multicolumn{2}{c|}{ Post program } & \multicolumn{2}{c|}{ after 6 months } \\
\cline { 2 - 7 } & \multicolumn{1}{|c|}{$\mathrm{R}$} & \multicolumn{1}{|c|}{$\mathrm{P}$} & \multicolumn{2}{c|}{$\mathrm{P}$} & $\mathrm{R}$ & $\mathrm{P}$ \\
\hline Sex & -0.12 & 0.1 & 0.02 & 0.8 & 0.05 & 0.7 \\
\hline Residents & 0.005 & 0.9 & 0.04 & 0.8 & 0.05 & 0.7 \\
\hline Age & 0.32 & 0.05 & -0.25 & 0.1 & -0.25 & 0.1 \\
\hline Years of experience: & 0.24 & 0.4 & -0.11 & 0.4 & -0.05 & 0.7 \\
\hline Qualification of nurses: & -0.20 & 0.2 & 0.25 & 0.1 & 0.03 & 0.8 \\
\hline occupation: & -0.33 & $0.04^{*}$ & 0.04 & 0.8 & 0.001 & 0.9 \\
\hline
\end{tabular}

Table (8) shows that there is no significance correlations between demographic characteristics and infection control practice among the studied participants, while for age and occupation there are mild correlation at pre, post and after 6 months of 
the education program. Meanwhile there are significance correlation regarding to occupation (p. $>0.05)$ at pre- program, as well as regarding to qualification of nurses there is mild significance correlation at six months post education program.

- There are no significance correlation between demographic characteristics and hemodialysis practice among the studied nurses, while regarding to age and years of experience are mild correlation at pre education.

- There are significance correlation regarding age and years of experience $(\mathrm{p} .<0.05)$.

- Regarding to qualification of nurses is mild correlation (r. 0.27) at post education program

\section{Discussion}

This chapter discussed the results of the current study, compares them with other related studies, recent literatures, as well as representing the researcher interpretations of some results. The aim of the current study was to study the effect of educational program about infection control precautions for nurses in pediatric hemodialysis units.

Patients with End Stage Renal Diseases (ESRD) are vulnerable to infectious diseases due to multiple comorbidities and reduced immune function. Due to the passive nature of dialysis therapy, hemodialysis patients can easily spread diseases through the narrow space between their beds. Therefore, appropriate infection control measures are very important to prevent the transmission of diseases in dialysis facilities (Hayne, Young-Ki ,2018).

The current study's results revealed that more than two thirds of the studied nurses were female, while less than one third were male, and their ages were more than 20 and less than 30 years. This may be due to our culture was against male registration in nursing program so registration in nursing school started at the 21 th country. This result was similar to demographics characteristics that were reported by Fawzy, (2016) in hemodialysis at Alexandria University hospital who found that more than half of the nurses were between 20 and 30 years, while EL-moghazy, (2013) found that most of the participants were female and the majority of them were more than 30 years.

This study's results were similar to demographic characteristics that reported by Basiony, (2014) in Beni-sueff hemodialysis hospitals who found that the majority of nurses were female and their ages less than 34 years. On the other hand Ali, (2013) found that the majority of the nurses' age ranged between twenty to fifty years and near to three quarters of them were female. Regarding male admission in nursing faculties the ratio is still limited compared by female, as well as in technical institutes.

According to Kadium, (2015) the proportion of male participants in his study was (7\%), which was comparable to the $(16.67 \%)$ reported by Deshmukh, \& Shinde, (2014), and $33.8 \%$ reported by Bianco , Coscarelli, Nobile, Pileggi, C., \&Pavia, (2013).

The present study showed that nearly one third of the participants had less than 5 years of experience and from 5 to less than 10 years of experience. It was detected that there were statistical significant relationships between nurses' overall practices and their years of experiences, as well as their qualifications and age. In other words, nurses with longer years of experience had better practice of infection prevention and control practices than the less experienced nurses.

These findings were congruent with Fawzy,(2016) who reported that nurses with longer years of experience had better practice of infection control precaution at HD units. These results may be attributed to day to day activities that enhances nurses' experience and improves their practice.
Nevertheless, Metwally, Abou Donia \& Abdel Aziz, (2016) indicated that nurses with 5 to less than 10 years of experience had higher mean percent practice score than those with more than 10 years of experience.

This finding is consistent with Basiony, (2014) in Beni-suff hemodialysis hospitals and Fawzy, (2016), Abdelfatah, (2013). Bakey,(2014), and Mohmmed, (2010) who reported highest percentages for the participants with less than 10 years' experience. However, the findings disagreed with Hassona. et al, (2011) who reported that more than half of the participants had more than 10years experience.

Regarding nursing qualification the present study's results showed that less than half of the studied nurses have diploma of secondary nursing school, while almost one third had technical institute of nursing.

These results were consistent with the reality that nursing secondary schools graduates represent the majority followed by technical institute of nursing then nursing faculties.

This finding goes in the same line with Basiony, (2014) in Beni-suef hemodialysis hospitals who found that all participants had technical nursing diploma, as well as Mohamed, (2008) who reported that all participants had diploma level of education; Fawzy, (2016), Ahmed, (2011) and Abdelfatah, (2013) in Assuit hospitals reported that less than half of nurses had diploma degree and less than one quarter of them had bachelor degree.

Hassona, et al, (2011) found that all participants had technical nursing diploma except one who has associate degree in nursing while Ali, (2013) found that more than three quarters of nurses had diploma level of education.

In addition, the study's results indicated that nurses with bachelor degree had significantly higher mean score of practice than those with diploma degree. This could be interpreted by the fact that bachelor degree nurses have more extensive undergraduate programs of infection control and microbiology than those with diploma of secondary nursing schools.

In the same context, Deghidi \& Growder , (2010) reported that education is a potential means for implementing prevention strategies as it alters perception, increases knowledge and in turn changes work practice.

Finding of the present study indicated that all of participants were attending unit staff development programs, orientation programs, or attending conference during the past five years affiliated to (ICU), (EMOH) and (WHO). this finding disagree with Basiony, (2014) in Benisuef hemodialysis hospitals who reported that none of nurses attending unit staff development programs, orientation programs or attending conference during the past five years and observed there was limited source of education activities for nurses in the study sitting. Meanwhile Abdelfatah, (2013) found that most of participants had no previous training.

The current study's showed that there are statistical significance differences between nurses' knowledge total 
scores at pre, post and after six months of education program (p.0.001). This results were in the same direction with Shrestha, (2014) who indicated that there was a significant difference between the pre intervention and post intervention knowledge score $(p=0.039)$, and this study showed that educational intervention program significantly improved the nurses' level of knowledge about the care of the patient with CVC.

On the other hand Abdelfatah, (2013) showed that there is no statistical significant difference between nurses' knowledge and their practice, and added that this may be attributed to insufficient courses related to hemodialysis included in their undergraduate curriculum of nursing education and also there is no available Arabic source for updating and continuing their education. This result agreed also with Hussein \& Mooji, (2010) who indicated that there is no significant difference between nurses' knowledge and their practice observed during the pre-test. As well as Abdelsatir, (2013) reported that there was no statistical significant difference, between pre and post program knowledge. The study suggested that HD units required organizing adequate training on HD access care for their nursing staff.

In relation to nurses' knowledge about infection control in hemodialysis, the result showed that the studied nurses had unsatisfactory knowledge about infection control preprogram and there are highly statistical significant in which increased knowledge scores post program conduction about infection, protection method and hand washing, precautions in hemodialysis.

This result was in the same line with AbdHady,Khalifa , Zein El-Dein. N.Hemdan, (2011) who indicated that there was a highly statistical significance difference between posttest, retention test than pretest and supported by Abdelsatir, (2013) who enhance the role of education in improving nurses knowledge. However, their practice still incompetent due to lack of facilities.

Regarding nurses, practice related to universal precaution of infection control the study's result revealed that there was an increase in knowledge score in all knowledge at post educational areas. In the same field Bayoumi\& Mahmoud, (2017) studied the effect of education program on nurses' knowledge and practice regarding care of central venous line in pediatric hemodialysis: evidencebased practice guidelines,

They found that there was a highly statistical significant improvement in nurses' practice such as hand washing, wearing mask, goggles, sterile gown, and gloves, use of disinfection, and dressing, when compared with that before and immediately after implementation and also before and 6 months after implementation of teaching guidelines.

In accordance with the study results, Abou El-Enein , ELMahdy, (2011) concluded that none of the nurses washed hands before and after the deferent activities that required hand washing or the use of plastic aprons or face protection among nurses in the dialysis unit in a University Hospital in Alexandria. On the other hand in a study done by Chenoweth, et al, (2015) observed patient care across hemodialysis facilitates enrolled in National Opportunity to Improve Infection Control in ESRD (end-stage renal disease) (NOTICE) project in order to evaluate adherence to evidence-based practices.

Many studies were agreed with Basiony, (2014). who found poor compliance to hand hygiene which might be attributed to lack of continuous and efficient in-service training and absence of supervision.

The current study's result showed that there were highly statistical significant differences in all of wearing gloves, administration of intramuscular, injection medication preparation, injection medication administration as pre and post educational program.

This result was consistent with Abd-ElHady, et al, (2011). who observed that the nurses know well the importance of hand washing for infection control, so more than two thirds of the nurses inadequately or infrequently performed and there were statistical significant differences between their performance on pretest, posttest and retention test. The author added that this result could be attributed to nurses used to rub hands with alcohol due to work overload in that area.

The current study's result illustrated that on pretest, the majority of nurses performed hand washing inadequately. This finding was consistent with National Health Service, (2014). that reported that hand washing is a complicated task comprising not only choice of appropriate disinfecting agent, but also frequency, duration, appropriateness, and liability for skin irritation from frequent washing. These findings were consistent with Dawood, (2011) who reported that lack of infection control equipment and supplies was one of the major factors to the application of precautions.

Regarding to nurses' practices in infection control precautions on starting hemodialysis procedure, it was found that there're high significant differences between pre and post program conduction ( $p$ valu.0.001), which might reflecting the effect of educational program. While decreased after six months. This result was supported by E Bagheban, SLakdizaji, Zamanzadeh \& Hasankhani, (2018). who concluded that the average score of knowledge and practices of the two groups before and after intervention was significantly different. Educational intervention increased knowledge $(\mathrm{P}=0.05)$ and practice in hand washing $(\mathrm{P}=$ $0.001)$, disinfection of the dialysis unit $(P=0.001)$, use of catheter $(\mathrm{P}=0.011)$, and needle insertion or removing of fistula $(\mathrm{P}=0.001)$ in the intervention group.

Our study's results indicated that more than two thirds of the studied nurses discard syringe in correct way in pre and post educational program, While in Abd-ElHady et al, (2011) showed that nurses' application of infection control precautions during dialysis, and at terminating dialysis as well as after session, only $(8.0 \%)$ of nurses discarded needles in sharp container on pre- test, all nurses applied sterile dressing on the shunt and wrapped it securely. Other studies done by Bakey,(2014), Karkar, Bouhaha, Dammang, (2014), Ebrahem, (2009) and Dawood, (2011) who reported that nurses threw out needles in leak-proof, puncture-resistant and color -coded boxes throughout all hemodialysis procedures. This could be attributed to lack of supervision.

For the nurses' practice about hemodialysis the current study revealed that the mean scores of the nurses' practice related to universal precaution of infection control pre/post educational program. This indicated the increase in practice mean score in all practice areas at the post program phase. However, this increase reached statistical significance for nurses' practice all of procedure are the same, about hand washing (p.0.001), gloving(p.0.001), while in AbdEl-Hady,et al., (2011) about the care of non-disposable equipment, removing mask and 
goggles, and sterile gowns, all nurses (100.0 \%) didn't perform these procedures due to the unavailability of these supplies. These findings were consistent with Ebrahem, (2009) and Dawood, (2011) who reported that lack of infection control equipment and supplies were the major obstacles to apply infection control properly.

Our study's results illustrated that the mean scores of the nurses' practice related to arteriovenous fistula graft cannulation and de-cannulation pre/post educational program indicated that there was highly statistical Significance differences between pre/post-educational program phases; no one of nurses done correctly performs hand hygiene before or after arteriovenous fistula graft cannulation. This result was in harmony with the study done by Ashour, (2016) Ramadan, (2016) and Hassona. et al, (2011) who reported that the highest percentage of their study nurses didn't comply with hand washing neither before nor after patients contact during IV fluid administration.

In the same context Erasmus et al (2010) concluded that Noncompliance with hand hygiene guidelines is a universal problem, which calls for standardized measures for research and monitoring. Theoretical models from the behavioral sciences should be used internationally and should be adapted to better explain the complexities of hand hygiene. Moreover Moursy \& Sharaf,(2017) indicated that the majority of the nurses were acquainted with the significance of hand washing, many nurses were carrying out hand hygiene inadequately in morning shifts and neglected it in the afternoon shifts either during cannulation or decannulation. This result could be referred to the lack of time, nurses' staff shortage and the workload in HD unit.

Personal protective equipment is compulsory to prevent cross infection. The present study showed that there were higher increases in percentages between steps done correctly pre/post educational programs phase.

However there are statistical significance differences between nurses' practice as pre/post educational programs. Moreover, the study results revealed that one third of the nurses choose the correct size glove in preprogram Wearing gloves are required whenever caring for a patient or touching patient's equipment. According to Bianco, et al., (2013) revealed that all nurses used only clean gloves instead of sterile ones during the whole shifts. This result may be attributed to the cost of sterile gloves, lack of strict policies and supervision, as well as workload.

According to CDC, (2010a) the use of sterile gloves during vascular access care is emphasized. In the same field Higgins\& Evans, (2011) reported that lack of infection control equipment and supplies were the main factor to the implementation of infection control practices.

Concerning care of vascular access site for prevention of infection, the present study indicated that only one who was apply skin antiseptic and allow it to dry in preprogram and there was highly statistical significance difference between pre and post educational program as regards to care of vascular access site correctly. As well in all procedures' steps it was observed that no one apply antimicrobial ointment in pre educational phase.

In the same context Ahmed et al, (2013). reported that the majority of the studied nurses in both shifts did not use to clean access arm for a minute with soap and water or antiseptic soap and placing access limb on a sterile drape or barrier was done inappropriately.

In this regard the National Health Service, (2014). Emphasized that the nurse should wash the access site with $P$ a g e $\mid 85$ soap and water then places the access limb on a sterile drape before cannulation to decrease the invasion of the micro flora into the blood stream. Bakey ,(2014). And Dawood, (2011) postulated that HD vascular access infections can be prevented by in service education and training programs for nurses, as they are largely responsible for the care of vascular access.

Our study's results showed that most of the nurses had poor knowledge about infection control pre educational program but post educational program the knowledge were at average level, meanwhile U.S. Renal Data System, USRDS, (2013). also showed that all nurses were not use the protective measures, in spite of the good knowledge of the majority. Also, the majority of them had poor knowledge about wearing goggles or face shields. This could be due to nurses' underestimation of the significance of these protective measures and the unavailability of these supplies.

The above findings were consistent with, Higgins \& Evans, (2011). Who reported that lack of infection control equipment and supplies was the main hindering factor to the implementation of infection control practices. In this context WHO, (2012). Recommended wearing Personal protective equipment when splashing, spraying or splattering of blood or body fluid is expected, and during HD vascular access cannulation or de-cannulation.

Regarding Routine disinfection of the Dialysis Station after patient has left station of the dialysis pre/post educational program which indicate that there are highly difference in percentage between nurses' practices who done correctly all procedures' steps among pre and post -educational program and observed that no one follow the basic step in infection control ( hand hygiene ). Although in Moursy \& Sharaf ,(2017)show that on pre-test, the majority of nurses $(80.0 \%)$ performed disinfection and rinsing for hemodialysis machine inadequately.

This finding was consistent with Bakey, (2014) who reported that nurses rinsed fluid pathway after every dialysis with dialox or citrosteril for 30 minutes. Also, this result consistent with Ebrahem , (2009) who reported that half of nurses disinfected external surfaces of hemodialysis machine.

In our study explain how the results support the hypothesis and how the explanation is consistent or fit in with previously and existing knowledge on the topic. And observed that knowledge and practice improve after educational program and start to reduce after 6 months which indicate that continues training and education will prevent the transmission of diseases in dialysis station

Therefore, nurses at HD units must possess an appropriate knowledge, skills and experience to prevent and control infection for themselves and for their patients and the continues training will improve the knowledge and skills

\section{Conclusion}

It can be concluded from the study's results that there were statistical significant relation between knowledge related to infection control pre, post and after 6 months of the educational Program. There were statistical significant relations between infection control practice pre, post and after 6 months of the program. The study's results indicated that there were improvement in control of infection in hemodialysis unites after educational program was applied. 


\section{Recommendations}

- Continuing education programs on regular basis is suggested in order to refresh and update nurse's knowledge،

- Written booklets, posters and videos should be available in each unit in hospital to acknowledge nurses about infection control procedures .

- Rules and regulation for hospital visitors and relatives must be settled and applied

- Provision of adequate resources, facilities and equipment related to any procedures in hemodialysis units such as eating, change clothes or child support.

- Repeated this research on large sample to ensure generalizability of the study.

\section{References}

1) Abd Elfatah A.H.A, (2013) Assessment of nursing knowledge and practice related to nursing care of children undergoing hemodialysis at assuit city, Unpublished Master thesis, Department of medical surgical nursing science, Faculty of Nursing .Cairo University.

2) Abd Elsatir. S,(2013) Evaluation of nurses' awareness and practice of hemodialysis access care in Khartoum state, Sudan. Arab Journal of nephrology and transplantations, 6(2), 119-121.

Retrieved from

(http://www.ajol.info/index.php/ajnt/article/view/88 699).

3) Abd-Hady Sh,Khalifa ,M .Zein El-Dein. N.Hemdan .H,(2011) Effect of an infection control competency based protocol on the occurrence of blood brone infection in hemodialysis unite at Minofiya university hospital Asian J.Med.pharm Res.1(1):2638.

4) Abd ElHafeez.S, Bolignano.D, D'Arrigo.D, Dounousi.E, Tripepi.D, and Zoccali.C ,(2018) Prevalence and burden of chronic kidney disease among the general population and high-risk groups in Africa: a systematic review, BMJ Open; 8(1): e015069.

5) Abou El-Enein ,Y\&ELMahdy.H,(2011) Standard precaution study among nurses in the dialysis unite in a University hospital in Alexandria Egypt .Journal of the Egyptian public health association ,86(1-2):3-10.

6) Ahmed G.H,(2011) Effect of designed nursing protocol on nurses 'knowledge and practice regarding hemodialysis patients, Unpublished Master thesis , Department of medical surgical nursing science, Faculty of Nursing .Assuit University.

7) Ahmed A.M.A., Allam M.F. Habil,E.S., A.M., Metwally N.A. Ibrahiem,M. Radwan, M.M. ElGaafary and Gadallah M.A, (2013) Compliance with haemodialysis practice guidelines in Egypt, Eastern Mediterranean Health Journal, Vol. 19 No.(1) pp. 4-9.

8) Ahamed.S.T\& Sallam.S.A,(2018) The Effect of Nursing Instructions on Nurses' Knowledge, Practice and Suggestions Regarding Adverse Events in Hemodialysis,
9) American Journal of Nursing Research, Vol. 6, No. 5, 237-243 Available online at http://pubs.sciepub.com/ajnr/6/5/4

10) Ali S.R, (2013) Assessment of nurses, knowledge and practice provided to the patients undergoing hemodialysis at Cairo hospitals Unpublished Master thesis, Department of medical surgical nursing science, Faculty of Nursing .Cairo University.

11) Ashour .M,(2016) Safe nursing practice applied for patient post hip joint replacement .unpublished master thesis faculty of nursing Alexandria university.

12) Association for professional's infection center, (2014) Guide to the Elimination of Infections in Hemodialysis. Available from http://www.apic.org/Resource [Last accessed on 16 April 2014] Back to cited text no. 22.

13) Bakey S.J, (2014) Evaluation of nurses' practices throughout hemodialysis treatment for patients in hemodialysis unites at Baghdad teaching hospitals .Kufa Journal for nursing science 2:2.

14) Basiony M.B,(2014) Staff nurses compliance with quality nursing care standers at hemodialysis units in selected hospitals at Beni-suef governorate. Unpublished Master thesis, Faculty of Nursing .Cairo University .

15) Bianco , A., Coscarelli, P., Nobile, C. G., Pileggi, C., \&Pavia. M, (2013) The reduction of risk in central line associated bloodstream infections: Knowledge, attitudes, and evidence-based practices in health care workers. American journal of infection control, 41(2), 107-112.

16) Bayoumi, M.H\& Mahmoud .N.F, (2017) Effect of education program on nurses' knowledge and practice regarding care of central venous line in pediatric hemodialysis: evidence-based practice. Egyptian Nursing Journal, enj.eg.net 14: 2, P: 8799.

17) Burns ,N.and Grove ,S,K,(2012) The practice of nursing research appraisal ,synthesis, and generation of evidence .St. Louis,Mo:Saunders Elsevier.

18) Centers for Disease Control and Prevention (CDC), (2010a) Healthcare-associated infections (HAI): guidelines and recommendations. Retrieved from http://www.cdc.gov.

19) Centers for disease control and prevention (CDC),(2010b) Ten leading cause death ,Retrieved from http://www.cdc.gov/injery /wizards/leading cuases.html.

20) Centers for Disease Control and Prevention(CDC), (2011) Guidelines for the prevention of intravascular catheter related infections. Retrieved from http://www.cdc.gov/hicpac/pdf/guidelines/bsiguidel ines- 2011.pdf.

21) Centers for Disease Control and Preventive (CDC), (2014) Hand Hygiene in Healthcare Settings Hand Hygiene Basics. Available http://www.cdc.gov/handhygiene/Basics.html.

22) Centers for Disease Control and Preventive (CDC), (2013) Infection Control, Frequently Asked Questions- Personal Protective Equipment (Masks, Protective Eyewear, Protective Apparel, Gloves) . 
23) Chenoweth,C.E,Hines,S,C,Hall,K,K,Saran,R,Kalbfl eisch,J.D.Spencer,t\&Messana,J.M,(2015) Variation in infection prevention practices in dialysis Facilities : Results From the National Opportunity to Improve infection Control in ESRD(End Stage Renal Disease) project .Infection Control \&Hospital Epidemiology.

24) Dawood .R,(2011) Effect of implementation protocol of post-operative arteriovenous fistula care on the clinical outcome of patients with end stage renal diseases .unpublished master's thesis .faculty of nursing .Alexandria university.

25) Deghidi S and Growder .D, (2010) Knowledge, awareness and compliance with universal precautions among health care workers at the university hospital of the West Indies, Jamaica. Theijoem: 1(4):171-8.

26) Deshmukh, M., \& Shinde. M, (2014) Impact of structured education on knowledge and practice regarding venous access device care among nurses. International Journal of Science and Research, 3(1), 895-901.

27) E Bagheban Karimi, SLakdizaji , V Zamanzadeh, H Hasankhani ,(2018) The Effects of Infection Control Teaching on the Knowledge and Performance of Hemodialysis Nurses in Tabriz, IJN 2018, 31(111): 1-9.

28) Ebrahem .G, (2009) Nursing Application of infection control precautions in Hemodialysis unit at El-Mansoura University Children's hospital. Unpublished Master in pediatric Nursing, Faculty of nursing University of Alexandria, 60-109.

29) Erasmus.V, Thea J. Daha, Hans Brug, Jan Hendrik Richardus ,(2010) Systematic Review of Studies on Compliance with Hand Hygiene Guidelines in Hospital Care, Infection Control \& Hospital Epidemiology Volume 31, (3), pp. 283294.

30) El-Moghazy G.E, (2013) Nursing knowledge and practices regarding intradialytic complications for hemodialysis patient. Journal of American Science, 9:11.

31) Emily, M.and Trish. M, (2011) Hospital epidemiology and infection control in acute care sittings .clinical microbiology reviews. vol24 (1).Pp.141:173.

32) Elsayed.E, El-Soreety.W, Elawany.T and Nasar.F ,(2012) Effect of nursing intervention on the Quality of life of children undergoing hemodialysis, Life Science Journal;9(1).

33) Fawzy .N, (2016) Infection control practices of nosocomial infection at hemodialysis unites, Unpublished Master thesis, Faculty of Nursing .Alexandry University.

34) Higgins,M and Evans .D,(2011) Nurses' knowledge and practices of vascular access infection control in hemodialysis patients in republic of Ireland, Journal of renal care .34(2):48-53.

35) Hussein. M, Mooji. J, (2010) Methods used to reduce the prevalence of Hepatitis $\mathrm{C}$ in a dialysis Unit. Saudi J Kidney Dis Transpl; 21: 909-13.

36) Hassona, F.M.H., Winkelman, C., El-Wahab, E.A.A., Ali S.R M.H., \& Abdeen, M.A, (2012) Evaluation of an educational program: A report from the Hemodialysis Unit in Zagazig University
Hospitals, Egypt. Nephrology Nursing Journal, 39(1), 53-59.

37) Hayne Cho Park, Young-Ki Lee, (2018) Korean clinical practice guidelines for preventing the transmission of infections in hemodialysis facilities. Kidney Res Clin Pract. 2018 Mar; 37(1): 8-19.

38) JaneW.Ball and Ruth C. Blinder ,( 2012) principles of pediatric nursing, care of renal disorder, 5 th ed , chapter 14 , pp. 1211.

39) Judith .A, Guzman .C and Karen. A, (2013) Infection prevention and control in residential facilities for pediatric. Infection control and hospital epidemiology .Cambridge university press.vol34 (10).pp. 1003:1041.

40) Karkar A, Bouhaha BM, Dammang MLA, Bouhaha BM, Dammang ML, (2014) Infection control in hemodialysis units: A quick access to essential elements. Saudi J Kidney Dis Transpl; 25:496-519.

41) Kadium, M, Jawad,(2015) Improving Nurses' Knowledge to Reduce Catheter-Related Bloodstream Infection in Hemodialysis Unit, Walden University Scholar Works, Project for the Degree of Doctor of Nursing Practice .

42) Lotfy E, Sakla N, El-Naggar S,(2015) Demographic study with risk factors of hemodialysis patients in Damietta Governorates, Egypt. International journal of Advanced Research . Vol 3, Issue 10, 91-100.

43) Metwally H, Abou Donia S, Abdel Aziz T,(2016) Safety nursing measures for patient undergoing gastrointestinal endoscopy . Alexandria scientific nursing journal: 18(1):17-41.

44) Mohamed. SH, (2018) Effect of accidence prevention program on the nursery school teacher's knowledge and practices. Unpublished doctorate thesis, Department of pediatric nursing science, Faculty of Nursing .El-mina University.

45) Mohamed. W, (2010) Prevention of Hepatitis C Virus in Hemodialysis Patients: Five Year Experience from a Single Center. Saudi J Kidney Dis Transpl; 21:548-54.

46) Mohamed E.M, (2008) Assessment of nurses' knowledge and performance regarding the nursing care given to chronic renal failure patient undergoing maintenance hemodialysis. Unpublished Master thesis, Department of medical surgical nursing science, Faculty of Nursing .Tanta University.

47) Mabrouke E.(2015) Assessment of hemodialysis adequacy in patients with chronic kidney disease in the hemodialysis unit at Tanta University Hospital in Egypt. Kasr Al-Ainy Medical Journal; 21(2): 4754.

48) Moursy A. \& Sharaf A.(2017) Vascular access care at hemodialysis unit; nurses compliance to infection prevention and control practices. IORS Journal of Nursing and Health Science. Mar -Apr.2017; Vol. 6 , Issue 2.

49) National Health Service, (2014) Competence framework for health care assistants .health care assistant competencies, Generic knowledge and skills .Retrieved from http: //www.HCA/competency framework.

50) Nicki L .Potts and Barbara .L,(2011) pediatric nursing caring for children and their families, 3rd ed, , chapter 11, pp822:896. 
51) Ramadan. S, (2016) Nurses compliance with slandered practices in intensive care units. Unpublished master's thesis, faculty of nursing .Alexandria University.

52) Shrestha. R, (2014) Impact of educational interventions on nurses' knowledge regarding care of the patient with central venous line. Journal of Kathmandu Medical College. Vol. 2. No. 1.Issue 3. Retrieved from http://www.jkmc.com.np/issue/3/28-30.pdf_br.

53) WHO,(2012) Save Lives: Clean Your Hands. Hand Hygiene in Outpatient and Home-Based Care and Long-Term Care Facilities. A guide to the application of the WHO multimodal hand hygiene improvement and the "My five moments of hand hygiene" approach. WHO Library Catologuing-inpublication Data. Available from: http://www.who.int/gpsc/5may/hh_guide.pdf. [Last accessed on 16 April 2014).

54) U.S. Renal Data System, USRDS, (2013) Annual Data Report: Atlas of Chronic Kidney Disease and End-Stage Renal Disease in the United States, National Institutes of Health, National Institute of Diabetes and Digestive and Kidney Diseases, Bethesda, MD. Retrieved from http://wwrw.usrds.org/adr.aspx 